Z Rheumatol 2020 · 79:686-691

https://doi.org/10.1007/s00393-020-00878-0

Online publiziert: 26. August 2020

(C) Der/die Autor(en) 2020

\section{Redaktion}

U. Müller-Ladner, Bad Nauheim

U. Lange, Bad Nauheim

J. Leipe ${ }^{1,2} \cdot$ B. F. Hoyer ${ }^{3} \cdot$ C. Iking-Konert ${ }^{4} \cdot$ H. Schulze-Koops ${ }^{2} \cdot$ C. Specker ${ }^{5}$.

K. Krüger ${ }^{6}$

'Sektion Rheumatologie, Medizinische Klinik V, Universitätsklinikum Mannheim, Mannheim, Deutschland

${ }^{2}$ Sektion Rheumatologie und Klinische Immunologie, Medizinische Klinik und Poliklinik IV, LudwigMaximilians-Universität München, München, Deutschland

${ }^{3}$ Abteilung für Rheumatologie, 1. Medizinische Klinik, Universitätskrankenhaus Schleswig-Holstein Campus Kiel, Kiel, Deutschland

${ }^{4}$ III. Medizinische Klinik und Poliklinik Sektion Rheumatologie, Universitätsklinikum Hamburg-Eppendorf, Hamburg, Deutschland

${ }^{5}$ Klinik für Rheumatologie und Klinische Immunologie, Kliniken Essen-Mitte, Essen, Deutschland

${ }^{6}$ Rheumatologisches Praxiszentrum München, München, Deutschland

\title{
SARS-CoV-2 \& Rheuma
}

\section{Konsequenzen der SARS-CoV-2-Pandemie für Patienten mit entzündlich rheumatischen Erkrankungen. Ein Vergleich der Handlungsempfehlungen rheumatologischer Fachgesellschaften und Risikobewertung verschiedener antirheumatischer Therapien}

Nachdem die Deutsche Gesellschaft für Rheumatologie e. V. (DGRh) bereits $\mathrm{zu}$ Beginn der COVID-19-Pandemie im März 2020 erste Handlungsempfehlungen zum Management von Patienten mit entzündlich rheumatischen Erkrankungen (ERE) unter dem Aspekt der SARSCoV-2/COVID-19-Bedrohung herausgegeben hat [13], wurden diese im Juli 2020 durch ein Update aktualisiert und erweitert (im Folgenden DGRh-Update) [14]. Während die initialen Empfehlungen in Ermangelung von Evidenz nahezu ausschließlich auf Expertenkonsens basierten, beruht das DGRh-Update auf der Datengrundlage von Registern, Querschnittstudien und Fallserien, welche bereits in kurzer Zeit wichtige Informationen zu SARS-CoV-2/COVID-19 bei ERE lieferten. Sämtliche dieser Datenquellen weisen Limitationen auf: Den wichtigsten potenziellen Bias stellt dabei u. a. die für diese Studienformen charak-

Die Autoren J. Leipe, B.F. Hoyer und C. Iking-Konert teilen sich die Erstautorenschaft. teristische überproportionale Erfassung schwerer Fälle dar; zudem auch das Fehlen von parallelen, regionalen Vergleichskohorten ohne ERE genauso wie der regional unterschiedliche Umgang mit SARS-CoV-2 bzw. die unterschiedliche Häufigkeit sowie die spezifischen Unterschiede der einzelnen Gesundheitssysteme.

Im DGRh-Update wurden Aspekte der Prävention bzw. des Managements von SARS-CoV-2/COVID-19, eine Risikoeinschätzung bei ERE sowie der Umgang mit immunmodulatorischen/ immunsuppressiven Medikamenten bei diesen Patienten thematisiert. Die Empfehlungen stimmen in vielen Punkten mit denen der amerikanischen (American College of Rheumatology [ACR]) [10] und europäischen (European League Against Rheumatism [EULAR]) [8] Fachgesellschaften überein, unterscheiden sich aber auch in einigen Punkten. Im Folgenden sollen daher Kernempfehlungen des DGRh-Updates im Kontext bzw. im Vergleich zu den ACR- und EULAR-
Empfehlungen näher beleuchtet und eine Übersicht zur Risikoeinschätzung einzelner antirheumatischer Medikamente gegeben werden.

\section{Prävention/Management von SARS-CoV-2/COVID-19}

Alle 3 Fachgesellschaften empfehlen die Einhaltung der seitens lokaler Behörden verordneten Verhaltens- und Vorsichtsmaßnahmen analog zur Allgemeinbevölkerung. Darüber hinausgehende Maßnahmen (z. B. der Gebrauch von FFP2/3Masken für besonders gefährdete Patienten) werden mangels wissenschaftlicher Evidenz nicht empfohlen. Unterschiede bestehen bezüglich Arztkontakten aufgrund der rheumatischen Erkrankung: Während ACR und EULAR bei kontrollierter Erkrankung und stabiler Therapie ausgewählte Maßnahmen zur Verringerung von persönlichen Kontakten - z. B. weniger Laborkontrollen, Nutzung von Telemedizin, verlängerte Dosierungsintervalle bei i.v.-Medikamenten und 
Verschiebung von rheumatologischen Vorstellungen - vorschlagen, wird von der DGRh eine normale Versorgung der Patienten mit ERE empfohlen. Dieser Unterschied ist sicher durch die momentan besser kontrollierte Infektionssituation in Deutschland gegenüber anderen europäischen Ländern und den USA zu erklären. Darüber hinaus zeigen neuere Veröffentlichungen, welche zum Zeitpunkt der Erstellung der ersten DGRhsowie der ACR- und EULAR-Empfehlungen größtenteils noch nicht vorlagen, dass kein generell erhöhtes Risiko für Patienten mit ERE besteht $[1,5-7,9$, 11]. Basierend darauf, überwiegt nun die Sorge vor einem potenziellen Schaden infolge (ungerechtfertigter) Einschränkungen der Versorgung („,medizinischer Kollateralschaden") gegenüber einem Infektionsrisiko durch Arztbesuche. Allerdings ist die Rückkehr zur normalen Versorgung in Deutschland auch an „Bedingungen“ geknüpft. So sollen Praxen und Ambulanzen entsprechende Verhaltens- und Hygienemaßnahmen sowie eine intelligente Sprechstundenplanung gewährleisten mit u. a. kürzeren Wartezeiten (weniger Patienten gleichzeitig in den Praxen und Ambulanzen), Einhalten nötiger Abstandsregeln und die maximale Reduktion der Zahl von Begleitpersonen, um Risiken $\mathrm{zu}$ minimieren. Zudem sollen die Patienten möglichst im Vorfeld systematisch abgefragt bzw. informiert werden, nicht mit Krankheitssymptomen von COVID19, nicht bei Rückkehr aus Risikogebieten (innerhalb der letzten 14 Tage; auch nicht nach initial negativem Testergebnis [16]) oder nach Kontakt zu nachweislich SARS-CoV-2-Infizierten in die Einrichtung zu kommen. Dies gilt auch für möglicherweise unabdingbare Begleitpersonen. Ausweichstrategien für Patienten mit Infusionsterminen in diesem Quarantänezeitraum sollten erarbeitet werden (ggf. stationäre Aufnahmen). In Hochrisikogebieten können striktere Verhaltens- und Vorsichtsmaßnahmen gelten.

\section{Risikoeinschätzung für Patienten mit entzündlich- rheumatischen Erkrankungen}

Bezüglich der Risikoeinschätzung für schwere COVID-19-Verläufe wurde in den Empfehlungen der DGRh, EULAR und ACR postuliert, dass Patienten mit ERE kein grundsätzlich erhöhtes Risiko einer Infektion mit SARS-CoV-2 oder eines schweren Verlaufes für COVID19 aufweisen. Vielmehr wurde in allen 3 Empfehlungen davon ausgegangen, dass das Risiko für einen schweren Verlauf in erster Linie mit den allgemeinen COVID-19-Risikofaktoren wie Alter und Komorbiditäten zusammenhängt [2]. Die deutsche Empfehlung geht, u.a. basierend auf teilweise kurz davor publizierten Daten, davon aus, dass auch die medikamentöse antirheumatische Therapie kein Risiko für einen schweren Verlauf von COVID-19 bei Patienten mit ERE darstellt, mit Ausnahme von Glukokortikoiden in einer Dosierung von $10 \mathrm{mg}$ Prednisolonäquivalent/Tag und mehr [4]. Die evidenzbasierte Risikoeinschätzung steht damit im Widerspruch $\mathrm{zu}$ der Bewertung des RKI, welche generell von einem erhöhten Risiko bei Einnahme von immunmodulatorischen Medikamenten ausgeht [17]. Bei der Einschätzung des RKI, die sich bereits seit Beginn der COVID-19-Krise auf der Webseite findet, handelt es sich vermutlich um eine Annahme; zudem ist sie, was die Bewertung unterschiedlicher Formen der Immunsuppression angeht, unpräzise. Unseres Erachtens sollte diskutiert werden, diese Angabe $\mathrm{zu}$ aktualisieren. Es ist jedoch nicht auszuschließen, dass zukünftige Daten noch (über das bei $>10$ mg Prednisolonäquivalent/Tag hinausgehende) weitere medikamentöse Risikofaktoren ergeben. Aufgrund der derzeit vorliegenden Daten erscheint es unwahrscheinlich, dass dies auf alle ERE-Patienten zutrifft. Einzelne Subgruppen könnten allerdings doch ein erhöhtes Risiko für schwere Verläufe von COVID-19 haben. Es scheint aktuell plausibel, dass das insgesamt niedrige Risiko unserer Patienten auch (und v. a.) an deren Umsichtigkeit im Umgang mit den empfohlenen Präventionsmaßnahmen liegt. Die Patienten sollten deshalb unbedingt angehalten werden, diese weiter konsequent durchzuführen.

Im DGRh-Update wurde, basierend auf aktuell publizierten Daten zu SARSCoV-2 und Erkenntnissen aus früheren Studien zum allgemeinen Infektionsrisiko, von einem erhöhten Risiko für einen schweren COVID-19-Verlauf bei unzureichend eingestellten ERE ausgegangen [3]. Basierend auf diesen Erkenntnissen, sollte daher bei Patienten mit gut eingestellter Erkrankung die antirheumatische Therapie nicht aus Sorge vor Komplikationen mit SARS-CoV-2 abweichend vom üblichen Vorgehen verändert oder pausiert werden. Jede Veränderung der laufenden Therapiebedeutet eine potenzielle Destabilisierung der Krankheitskontrolle.

\section{Umgang mit immunmodulato- rischen/immunsuppressiven Medikamenten}

\section{Antirheumatische Therapie bei Patienten ohne Infektzeichen}

Das DGRh-Update unterscheidet zwischen bestehender und Neubeginn/ Umstellung einer antirheumatischen Therapie. Bei bestehender Therapie sollen nichtsteroidale Antirheumatika (NSAR), Glukokortikoide (GC, <10 mg/ Tag Prednisolonäquivalent), „conventional synthetic disease modifying antirheumatic drugs" (csDMARDs), „targeted synthetic DMARDs“ (tsDMARD), „biological DMARDs“ (bDMARDs) und Immunsuppressiva unverändert fortgesetzt werden. Ähnlich empfiehlt auch die EULAR, NSAR, GC und DMARDs unverändert zu belassen, und die ACRTaskforce, NSAR, GC ( $\leq 10 \mathrm{mg} / \mathrm{Tag}$ Prednisolonäquivalent), DMARDs und Immunsuppressiva fortzusetzen.

Bei notwendigem Neubeginn/Umstellung einer antirheumatischen Therapie sollte gemäß DGRh-Empfehlung eine Behandlung nicht aufgrund der COVID19-Pandemie unterbleiben, verändert, verzögert oder unterdosiert werden. Generell sollte nach guter klinischer Praxis gemäß den aktuell geltenden Leitlinien für die entsprechenden Krankheitsbilder behandelt werden. Bei bestehenden Alternativen kann erwogen werden, eher 
Substanzen mit kurzer Halbwertszeit zum Einsatz zu bringen. Eine Präferenz für eine besondere Substanz oder Substanzklasse sprechen die Empfehlungen nicht aus. Es sollte aber - wie bisher - auf den unnötigen Einsatz von hoch dosierten Glukokortikoiden verzichtet werden.

Die ACR-Taskforce unterscheidet bezüglich des Neubeginns der Therapie noch zwischen Patienten mit SLE, Arthritiden und anderen ERE, obgleich angemerkt werden muss, dass die Empfehlungen $\mathrm{zu}$ diesen spezifischen Situationen noch mehrheitlich Expertenmeinungen darstellten und $\mathrm{zu}$ dem damaligen Zeitpunkt wenig evidenzbasiert waren. Demnach sollen Antimalariamittel unverändert bei neu diagnostiziertem systemischem Lupus erythematodes (SLE) in voller Dosis begonnen und bei Schwangeren mit gleicher Dosis fortgeführt werden; wenn indiziert, kann Belimumab initiiert werden. Patienten mit Arthritis sollen gemäß ACR, sofern gut kontrolliert, unverändert mit Antimalariamitteln und IL-6R-Inhibitoren (IL-6Ri) weiterbehandelt werden. Bei aktiver oder neu diagnostizierter Arthritis können csDMARDs begonnen oder umgestellt werden. Bei moderater bis hoher Krankheitsaktivität trotz csDMARD-Therapie können Biologika gestartet werden.

Speziell zu GC empfehlen DGRh und ACR, diese in der niedrigstmöglichen Dosis zur Kontrolle rheumatischer Erkrankungen einzusetzen, möglichst $\leq 10 \mathrm{mg}$ Prednisolonäquivalent/Tag. Eine GC-Dauertherapie < $10 \mathrm{mg} /$ Tag Prednisolonäquivalent sollte in gleicher Dosis fortgesetzt und moderat hoch dosierte und/oder niedrig dosierte Glukokortikoide sollten nicht abrupt abgesetzt werden, unabhängig von der SARS-CoV-2-Exposition oder dem Infektionsstatus. Zudem können selbst nach SARS-CoV-2-Exposition hoch dosierte Glukokortikoide im Kontext schwerer, vital organbedrohlicher Manifestation der rheumatischen Erkrankung (z.B. Lupusnephritis oder Vaskulitis) notwendig sein.

Z Rheumatol 2020 · 79:686-691 https://doi.org/10.1007/s00393-020-00878-0

(c) Der/die Autor(en) 2020

J. Leipe - B. F. Hoyer · C. Iking-Konert · H. Schulze-Koops · C. Specker · K. Krüger

SARS-CoV-2 \& Rheuma. Konsequenzen der SARS-CoV-2-Pandemie für Patienten mit entzündlich rheumatischen Erkrankungen. Ein Vergleich der Handlungsempfehlungen rheumatologischer Fachgesellschaften und Risikobewertung verschiedener antirheumatischer Therapien

\section{Zusammenfassung}

Die Empfehlungen des Deutschen Gesellschaft für Rheumatologie(DGRh)-Updates - welches die zu Beginn der COVID-19Pandemie erstellte Hilfestellung zum Management von Patienten mit entzündlich rheumatischen Erkrankungen angesichts der Bedrohung durch SARS-CoV-2 aktualisiert und erweitert - stimmen in vielen Punkten mit den Handlungsempfehlungen der amerikanischen (ACR) und europäischen Fachgesellschaften (EULAR) überein, unterscheiden sich aber auch in einigen Punkten. In diesem Artikel sollen daher Kernempfehlungen des DGRh-Updates zu den
Themen Prävention von SARS-CoV-2/COVID19, Risikoeinschätzung bei ERE sowie der Umgang mit antirheumatischen Therapien im Kontext bzw. im Vergleich zu den ACR- und EULAR-Empfehlungen diskutiert und eine Übersicht zur Risikobeurteilung einzelner antirheumatischer Medikamente gegeben werden.

Schlüsselwörter

COVID-19 - Management · Empfehlungen . Entzündlich rheumatische Erkrankungen . DMARD

\section{SARS-CoV-2 \& rheumatic disease. Consequences of the SARS-CoV- 2 pandemic for patients with inflammatory rheumatic diseases. A comparison of the recommendations for action of rheumatological societies and risk assessment of different antirheumatic treatments}

\section{Abstract}

The recommendations of the German Society of Rheumatology (DGRh) update, which update and expand the guidance on the management of patients with inflammatory rheumatic diseases in view of SARS-CoV-2 created at the beginning of the COVID19 pandemic, correspond in many points with the recommendations for action of the American (ACR) and European (EULAR) societies, but also differ in some points. Therefore, this article discusses the core recommendations of the DGRh update on the prevention of SARS-CoV-2/COVID-19, the risk assessment for inflammatory rheumatic diseases and the use of antirheumatic treatments in the context and in comparison to the ACR and EULAR recommendations, and provides an overview of the risk assessment of individual antirheumatic drugs.

Keywords COVID-19. Management $\cdot$ Recommendations . Inflammatory rheumatic diseases - DMARD

\section{Antirheumatische Therapie bei Patienten mit Kontakt zu SARS- CoV-2-positiven Individuen und ohne eigene COVID-19- Infektzeichen}

Hier empfiehlt die DGRh zunächst eine Fortführung der Therapie und die Kontaktaufnahme mit dem Rheumatologen bei Auftreten von Symptomen. Das ACR empfiehlt dagegen bereits in dieser Konstellation das Pausieren von Immunsup- pressiva, nicht aber von IL-6Ri-Biologika und JAK-Inhibitoren (JAKi), temporär bis zum Vorliegen eines negativen COVID-19-Tests oder nach 2 Wochen symptomfreiem Beobachtungszeitraum. Unter bestimmten Umständen, z. B. bei Riesenzellarteriitis, können gemäß ACR IL-6Ri fortgeführt werden. Die Empfehlung, IL6Ri und JAKi nicht abzusetzen, beruht auf der Annahme, dass eine immunmodulierende Therapie einen sog. „Zytokin-Sturm“ und dadurch schwerste 
Tab. 1 Übersicht über die momentane Einschätzung zu den einzelnen antirheumatischen Therapien und SARS-CoV-2/COVID-19 bei Patienten mit ERE $[3-7,9,11,12]$

\begin{tabular}{|c|c|c|}
\hline $\begin{array}{l}\text { Substanzklassen/ } \\
\text { Substanzen }\end{array}$ & $\begin{array}{l}\text { Effekt auf } \\
\text { COVID-19- } \\
\text { Verlauf }\end{array}$ & Sonstige Bemerkungen \\
\hline NSAR & $\Rightarrow$ & Initial diskutiertes Risiko nicht belegt \\
\hline \multicolumn{3}{|l|}{ CSDMARD } \\
\hline Hydroxychloroquin & $\Rightarrow$ & $\begin{array}{l}\text { Initial diskutierter protektiver Effekt aktuell } \\
\text { nicht belegt (Zitat u.a. Cochrane) }\end{array}$ \\
\hline Sulfasalazin & $\Rightarrow$ & - \\
\hline Methotrexat & $\Rightarrow$ & - \\
\hline Leflunomid & $\Rightarrow$ & - \\
\hline $\begin{array}{l}\text { Mycophenolat-Mofetil/ } \\
\text { Azathioprin/ } \\
\text { Calcineurininhibitoren }\end{array}$ & $\varnothing$ & - \\
\hline \multicolumn{3}{|l|}{ bDMARD } \\
\hline Abatacept & $\Rightarrow$ & $\begin{array}{l}\text { Potenziell eingeschränkte Impfantwort bei } \\
\text { COVID-19-Impfung? }\end{array}$ \\
\hline Belimumab & $\Rightarrow$ & - \\
\hline IL-1-Inhibitoren & $\Rightarrow$ & $\begin{array}{l}\text { Protektive Wirkung bei sehr schweren Verläu- } \\
\text { fen beschrieben } \\
\text { RCTs zum Einsatz bei COVID-19 laufen }\end{array}$ \\
\hline IL-6R-Inhibitoren & $\Rightarrow$ & $\begin{array}{l}\text { Protektive Wirkung bei sehr schweren Verläu- } \\
\text { fen in Fallserien } \\
\text { Zahlreiche RCTs zum Einsatz bei COVID-19 } \\
\text { laufen. Die ersten beiden berichteten Pha- } \\
\text { se-III-Studien (NCT04320615, NCT04315298) } \\
\text { ohne signifikanten Effekt }\end{array}$ \\
\hline IL-12/23-Inhibitoren & $\Rightarrow$ & - \\
\hline IL-17-Inhibitoren & $\Rightarrow$ & - \\
\hline Rituximab & $\pi$ & $\begin{array}{l}\text { Einzelfallberichte mit kritischem Outcome. } \\
\text { Potenziell eingeschränkte Impfantwort bei } \\
\text { COVID-19-Impfung? }\end{array}$ \\
\hline TNF-Inhibitoren & $\Rightarrow$ & $\begin{array}{l}\text { Protektive Wirkung diskutiert } \\
\text { RCTs zum Einsatz bei COVID-19 laufen }\end{array}$ \\
\hline \multicolumn{3}{|l|}{ tsDMARD } \\
\hline Apremilast & $\Rightarrow$ & - \\
\hline JAK-Inhibitoren & $\Rightarrow$ & $\begin{array}{l}\text { RCTs zum Einsatz bei schwerem COVID-19 } \\
\text { laufen }\end{array}$ \\
\hline \multicolumn{3}{|c|}{$\begin{array}{l}\text { Risiko für schweren Verlauf von COVID-19 aus wissenschaftlichen Daten: keine Hinweise für erhöhtes } \\
\text { Risiko: } \nearrow \text {, einzelne Fälle von schweren Verläufen: } \Rightarrow \text {, keine Daten oder nur wenige Fallberichte: } \varnothing \\
\text { aOR adjustierte Odds Ratio, bDMARD "biologic disease modifying anti-rheumatic drugs" } \\
\text { (bDMARDs), csDMARDs ",conventional synthetic DMARDs", RCTs "randomized controlled trials", } \\
\text { tsDMARD "targeted synthetic DMARDs" }\end{array}$} \\
\hline
\end{tabular}

Verläufe von COVID-19 ggf. verhindern kann. Insbesondere für Tocilizumab gab es schon früh Einzelberichte und Fallserien, dass sich u. a. die Notwendigkeit und Dauer von Beatmungspflichtigkeit, aber auch die Mortalität möglicherweise senken lässt [2]. Die nun kürzlich zunächst in Pressemitteilungen veröffentlichen ersten Informationen zur PhaseIII-Studie von Tocilizumab und Sarilumabkonnten diese Erwartung aber bisher nicht erfüllen [18, 19]; weitere Phase-III-
Antirheumatische Therapie bei Patienten mit Kontakt zu SARSCoV-2-positiven Individuen und mit eigenen COVID-19Infektzeichen

Hier empfiehlt die DGRh bei leichten Symptomen und fehlendem Fieber keine Therapieänderung, aber eine Kontaktaufnahme mit dem Rheumatologen und bei eindeutigen Infektzeichen, insbesondere Fieber $\left(>38^{\circ} \mathrm{C}\right)$, eine Pausierung der antirheumatischen Medikation. Zu dieser speziellen Konstellation nehmen weder EULAR noch ACR dezidiert Stellung.

\section{Antirheumatische Therapie bei positiv auf SARS-CoV-2 getesteten (PCR) Patienten ohne COVID-19- Infektzeichen}

Für diese Konstellation empfiehlt die DGRh ein Pausieren oder Herauszögern einer ts- oder bDMARD-Therapie für die Dauer der mittleren Inkubationszeit. Bei Symptomfreiheit sollte noch eine Pause für 5 bis 6 Tage nach Abstrich erwogen werden; csDMARDs sollten nicht abgesetzt werden.

\section{Antirheumatische Therapie bei positiv auf SARS-CoV-2 getesteten (PCR) Patienten mit COVID-19- Infektzeichen}

Für diese Konstellation einer COVID-19Erkrankung empfiehlt die DGRh ein Pausieren der DMARD-Therapie. Bei Einnahme von Leflunomid sollte wegen der langen Halbwertszeit ein Auswaschen erwogen werden, wobei dies angesichts fehlender Sicherheitssignale in COVID-19Registern und möglicher (derzeit in Studien getesteter) positiver Wirkung des Metaboliten Teriflunomid bei COVID19 durchaus diskussionswürdig ist.

Bei dokumentierter oder vermuteter COVID-19-Erkrankung empfiehlt das ACR in ähnlicher Weise Sulfasalazin, Methotrexat, Leflunomid, Immunsuppressiva, Nicht-IL-6Ri-Biologika und JAKi zu pausieren (Begründung s. oben), jedoch Antimalariamittel fortzusetzen. Letzteres wurde mangels überzeugender Daten zur zunächst erhofften positiven Wirkung von Hydroxychloroquin 
(HCQ) auf den Verlauf von COVID-19 seitens der DGRh nicht explizit empfohlen.

\section{Risiko antirheumatischer Therapie bei COVID-19}

Eine Übersicht über die momentane Einschätzung zu den einzelnen Substanzen/ Substanzklassen ist in - Tab. 1 zusammengefasst. Diese Einschätzung basiert auf der Literatursuche, die im Rahmen der Erstellung des DGRh-Updates durchgeführt wurde.

$\mathrm{Ob}$ sich möglicherweise sogar günstige Effekte von Glukokortikoiden, bDMARDs und tsDMARDs auf den Verlauf von COVID-19 bei Patienten mit ERE und/oder ohne ERE aus randomisiert kontrollierten Studien ergeben, wird sich zukünftig zeigen. Wie oben diskutiert, haben sich die zunächst hoffnungsvollen Daten u.a. zu HCQ und IL-6Ri in weiteren Studien nicht erfüllt, während es für eine Intervention mit Glukokortikoiden positive Signale gibt [15].

\section{Fazit für die Praxis}

Die vorgestellten Empfehlungen, v. a. das DGRh-Update, kommen nach Bewertung der vorliegenden wissenschaftlichen Daten zu der vorläufigen Schlussfolgerung, dass für Patienten mit ERE kein generell erhöhtes Risiko für die Häufigkeit oder einen schweren Verlauf einer COVID-19-Erkrankung besteht, es sei denn, es liegen der allgemeinen Bevölkerung entsprechende Risikobegleiterkrankungen, eine erhöhte Krankheitsaktivität oder eine Glukokortikoideinnahme von mehr als $10 \mathrm{mg}$ Prednisolonäquivalent/Tag vor. Auf der Basis dieser Daten empfiehlt die DGRh im Vergleich zu den Empfehlungen der EULAR und des ACR eine weniger restriktive Haltung bezüglich einer Vermeidung von Arztkontakten. Basistherapien sollten - im Gegensatz zu EULAR und ACR - nur beim Vorliegen eindeutiger Infektsymptome oder einem positiven SARS-CoV-2-Nachweis pausiert werden.

Bezüglich der Bewertung der einzelnen Substanzen ist an dieser Stelle noch ein- mal zu betonen, dass die Datenbasis für die Empfehlungen aus Fallserien, Kohortenstudien und Fall-Kontroll-Studien besteht und der Evidenzgrad somit noch begrenzt ist. Dies und Confounder, die nur begrenzt etwas mit der Substanz oder der rheumatischen Grunderkrankung zu tun haben, erschweren eine endgültige Bewertung des Risikos für schwere COVID-19-Verläufe. So werden bestimmte Substanzen vorrangig bei Patienten mit bestimmtem Vor-/ Begleiterkrankungen, wie z. B. Malignomen oder Herzinsuffizienz, eingesetzt, was hier zu einer Verzerrung führen kann. Auch können wir nicht abschließend beurteilen, inwiefern die aktuellen Daten zu Häufigkeit und Schwere von COVID-19 bei Patienten mit ERE durch ein besonders vorsichtiges Verhalten und eine restriktive Umsetzung der Präventionsmaßnahmen vonseiten unserer Patienten gegenüber der Normalbevölkerung begründet ist. Diese Faktoren limitieren zwar die Qualität aller Empfehlungen, trotzdem kann an dieser Stelle zumindest ein vorsichtiges Signal der Entwarnung gegenüber der ganz initialen Sorge um unsere $\mathrm{Pa}$ tienten gegeben werden, dass sie per se oder durch die spezifische Therapie einem höheren Risiko für eine SARSCoV-2-Infektion oder einem schweren Verlauf einer COVID-19-Erkrankungen ausgesetzt seien. Zukünftig ist zu erwarten, dass DGRh, ACR und EULAR, basierend auf neuen wissenschaftlichen Daten (auch eigener klinischer Register), weitere konkretere Handlungsempfehlungen veröffentlichen.

\section{Korrespondenzadresse}

\section{Dr. J. Leipe}

Sektion Rheumatologie, Medizinische Klinik V, Universitätsklinikum Mannheim Theodor-Kutzer-Ufer 1-3, 68167 Mannheim, Deutschland jan.leipe@umm.de

Funding. Open Access funding provided by Projekt DEAL.

Interessenkonflikt. J.Leipe, B.F. Hoyer, C. IkingKonert, H. Schulze-Koops, C. Specker und K. Krüger geben an, dass kein Interessenkonflikt besteht.

Open Access. Dieser Artikel wird unter der Creative Commons Namensnennung 4.0 International Lizenz veröffentlicht, welche die Nutzung, Vervielfältigung Bearbeitung, Verbreitung und Wiedergabe in jeglichem Medium und Format erlaubt, sofern Sie den/die ursprünglichen Autor(en) und die Quelle ordnungsgemäß nennen, einen Link zur Creative Commons Lizenz beifügen und angeben, ob Änderungen vorgenommen wurden.

Die in diesem Artikel enthaltenen Bilder und sonstiges Drittmaterial unterliegen ebenfalls der genannten Creative Commons Lizenz, sofern sich aus der Abbildungslegende nichts anderes ergibt. Sofern das betreffende Material nicht unter der genannten Creative Commons Lizenz steht und die betreffende Handlung nicht nach gesetzlichen Vorschriften erlaubt ist, ist für die oben aufgeführten Weiterverwendungen des Materials die Einwilligung des jeweiligen Rechteinhabers einzuholen.

Weitere Details zur Lizenz entnehmen Sie bitte der Lizenzinformation auf http://creativecommons.org/ licenses/by/4.0/deed.de.

\section{Literatur}

1. Aries $P$, Iking-Konert C (2020) No increased rate of SARS-CoV-2 infection for patients with inflammatory rheumatic diseases compared with the general population in the city of Hamburg (Germany). Ann Rheum Dis. https://doi.org/10. 1136/annrheumdis-2020-218400

2. Cortegiani A, Ippolito M, Greco M et al (2020) Rationale and evidence on the use of tocilizumab in COVID-19: a systematic review. Pulmonology. https://doi.org/10.1016/j.pulmoe.2020.07.003

3. D'silva KM, Serling-Boyd N, Wallwork R et al (2020) Clinical characteristics and outcomes of patients with coronavirus disease 2019 (COVID-19) and rheumatic disease: a comparative cohort study from a US 'hot spot'. Ann Rheum Dis. https://doi. org/10.1136/annrheumdis-2020-217888

4. Damiani G, Pacifico A, Bragazzi NL et al (2020) Biologics increase the risk of SARS-CoV-2 infection and hospitalization, but not ICU admission and death: Real-life data from a large cohort during red-zone declaration. Dermatol Ther. https://doi. org/10.1111/dth. 13475

5. Gianfrancesco M, Hyrich KL, Al-Adely S et al (2020) Characteristics associated with hospitalisation for COVID-19 in people with rheumatic disease: data from the COVID-19 Global Rheumatology Alliance physician-reported registry. Ann Rheum Dis 79:859-866. https://doi.org/10.1136/ annrheumdis-2020-217871

6. Gianfrancesco MA, Hyrich KL, Gossec L et al (2020) Rheumatic disease and COVID-19: initial data from the COVID-19 Global Rheumatology Alliance provider registries. Lancet Rheumatol. https://doi. org/10.1016/S2665-9913(20)30095-3

7. Haberman R, Axelrad J, Chen A et al (2020) Covid-19 in Immune-Mediated Inflammatory Diseases-Case Series from New York. N Engl J Med 383:85-88. https://doi.org/10. 1056/NEJMc2009567

8. Landewe RB, Machado PM, Kroon F et al (2020) EULAR provisional recommendations for the management of rheumatic and musculoskeletal diseases in the context of SARS-CoV-2. Ann Rheum Dis 79:851-858. https://doi.org/10.1136/ annrheumdis-2020-217877

9. Michelena $X$, Borrell $H$, Lopez-Corbeto $M$ et al (2020) Incidence of COVID-19 in a cohort of adult and paediatric patients with rheumatic diseases 
treated with targeted biologic and synthetic disease-modifying anti-rheumatic drugs. Semin Arthritis Rheum 50:564-570. https://doi.org/10. 1016/j.semarthrit.2020.05.001

10. Mikuls TR, Johnson SR, Fraenkel L et al (2020) American College of Rheumatology guidance for the management of rheumatic disease in adult patients during the COVID-19 pandemic: version 1 Arthritis Rheumatol. https://doi.org/10.1002/art. 41301

11. Monti S, Balduzzi S, Delvino P et al (2020) Clinical course of COVID-19 in a series of patients with chronic arthritis treated with immunosuppressive targeted therapies. Ann Rheum Dis 79:667-668. https://doi.org/10.1136/ annrheumdis-2020-217424

12. Pablos JL, Abasolo L, Alvaro-Gracia JM et al (2020) Prevalence of hospital PCR-confirmed COVID19 cases in patients with chronic inflammatory and autoimmune rheumatic diseases. Ann Rheum Dis. https://doi.org/10.1136/annrheumdis-2020217763

13. Schulze-Koops H, Holle J, Moosig F et al (2020) Current guidance of the German Society of Rheumatology for the care of patients with rheumatic diseases during the SARS-CoV-2/Covid 19 pandemic. ZRheumatol 79:385-388

14. Schulze-Koops H, lking-Konert C, Leipe J et al (2020) Recommendations of the German Society for Rheumatology for management of patients with inflammatory rheumatic diseases in the context of the SARS-CoV-2/COVID-19 pandemic-Update July 2020. Z Rheumatol. https://doi.org/10.1007/s00393-020-00799-y

15. Wu R, Wang L, Kuo HD et al (2020) An update on current therapeutic drugs treating COVID-19. Curr Pharmacol Rep. https://doi.org/10.1007/s40495020-00216-7

16. Bundesministerium für Gesundheit (2020) Regelungen für nach Deutschland Einreisende im Zusammenhang mit Coronavirus SARS-CoV-2 / COVID-19. https://www. bundesgesundheitsministerium.de/fileadmin/ Dateien/3_Downloads/C/Coronavirus/BMG_ Infoblatt_fuer_Einreisende_060820.pdf. Zugegriffen: 17. Aug. 2020

17. RKI (2020) SARS-CoV-2 Steckbrief zur CoronavirusKrankheit-2019 (COVID-19). https://www.rki.de/ DE/Content/InfAZ/N/Neuartiges_Coronavirus/ Steckbrief.html.Zugegriffen: 17. Aug. 2020

18. Roche (2020) Roche provides an update on the phase III COVACTA trial of Actemra/RoActemra in hospitalised patients with severe COVID19 associated pneumonia. https://www.roche. com/media/releases/med-cor-2020-07-29.htm Zugegriffen: 17. Aug. 2020

19. Sanofi Sanofi and Regeneron provide update on Kevzara ${ }^{\circledR}$ (sarilumab) Phase 3 U.S. trial in COVID19 patients. https://www.sanofi.com/en/mediaroom/press-releases/2020/2020-07-02-22-3000.Zugegriffen:17. Aug. 2020

\section{Fallpauschalen gefährden medizinische Versorgung von Menschen mit Rheuma}

DGRh kritisiert Schieflage im Abrechnungssystem

Vier bis sechs Wochen - länger sollte es nicht dauern, bis ein Patient mit Anzeichen für eine entzündlich-rheumatische Erkrankung einem Facharzt vorgestellt wird und die richtige Diagnose erhält. Dieses Ziel wird in Deutschland jedoch weit verfehlt. Ein Grund hierfür ist der ausgeprägte Mangel an rheumatologischen Fachärzten. Nach Ansicht der Deutschen Gesellschaft für Rheumatologie e.V. (DGRh) hat dieser inzwischen ein Ausmaß erreicht, welches die medizinische Versorgung der Bevölkerung gefährdet.

1,5 Mio. Menschen in Deutschland leiden an entzündlich-rheumatischen Erkrankungen, etwa zwei Prozent der erwachsenen Bevölkerung. Und vermutlich gibt es viele weitere, die noch nichts von ihrer Erkrankung wissen. Denn selbst eine rheumatoide Arthritis (RA) - mit 60 bis 70000 Neuerkrankungen pro Jahr die häufigste und bekannteste Rheumaart - wird im Schnitt erst nach neun Monaten diagnostiziert. Patienten mit selteneren Rheumaformen müssen noch deutlich länger, zum Teil mehrere Jahre auf Diagnose und Therapie warten. „Für die Betroffenen kann das gravierende Folgen haben", mahnt Prof. Dr. Hanns-Martin Lorenz, Leiter der Sektion Rheumatologie am Universitätsklinikum Heidelberg und medizinisch-wissenschaftlicher Leiter des Rheumazentrums Baden-Baden. Betroffene müssten nicht nur unnötig lange unter den Symptomen ihrer entzündlich-rheumatischen Erkrankung leiden, die fast immer mit Schmerzen und Abgeschlagenheit, manchmal auch mit einer Depression einhergehe. Auch der weitere Krankheitsverkauf hänge wesentlich davon $a b$, wie rasch mit einer wirksamen Therapie begonnen werde. Werde die Entzündung nicht rasch unter Kontrolle gebracht, sei die Gefahr groß, dass sie chronisch werde und zu irreversiblen Schäden führe. Umgekehrt könnten viele entzündlich-rheumatische Erkrankungen mit einer frühen und konsequenten Therapie gut beherrscht werden.

\section{Zahl internistischer Rheumatologen ist} zu verdoppeln

Hierfür stehen in Deutschland jedoch zu wenige Fachärzte zur Verfügung. „Um eine gute Versorgung zu gewährleisten, bräuchten wir mindestens 1350 internistische Rheumatologen", sagt Lorenz. Zurzeit liege deren Zahl mit 750 etwa bei der Hälfte. Um hier zumindest mittelfristig gegenzusteuern, müsste die Zahl der rheumatologischen Weiterbildungsstellen deutlich erhöht werden. Eine Hürde hierfür ist das 2004 eingeführte DRG-Abrechnungssystem, bei dem die klinische Patientenversorgung über Fallpauschalen vergütet wird. Diese richten sich nach Art und Schwere der Erkrankung, spiegeln jedoch nicht immer den tatsächlichen Therapieaufwand wider und sorgen dafür, dass manche Patienten sich für die Klinik eher "lohnen“ als andere. „Durch die Fallpauschalen zerfällt die Klinik in finanzkräftige und weniger finanzkräftige Abteilungen“, kritisiert Lorenz - „und damit in Abteilungen, denen mehr und anderen, denen weniger Weiterbildungsstellen zugeteilt werden.“ „Die Rheumatologie als eher ambulant ausgerichtete Fachdisziplin, die im Vergleich keine großen Umsätze generiert, bleibt hier systematisch unterversorgt", betont auch DGRh-Präsident Prof. Dr. Hendrik Schulze-Koops vom Klinikum der Universität München.

\section{Medizinische Versorgung nach Bedarf der Bevölkerung}

Die DGRh fordert daher, dass künftig eine Mindestzahl von Weiterbildungsstellen in der Rheumatologie garantiert wird. „Die Schwerpunkte in den Krankenhäusern dürfen nicht nur nach finanziellen Gesichtspunkten gesetzt werden, sondern hauptsächlich nach dem Bedarf in der Bevölkerung", so Lorenz. Nur dann könne eine breit aufgestellte medizinische Versorgung auf hohem Niveau gewährleistet werden.

\section{S. Priester, Pressestelle DGRh}

Deutscher Rheumatologie Kongress 2020 - virtuell 9. bis 12. September 2020 https://www.dgrh-kongress.de 